\title{
Chronic allergic fungal sinusitis invading the skull base in an immunocompetent male: illustrative case
}

\author{
Anant Naik, BS, ${ }^{1}$ Darrion Bo-Yun Yang, BS, ${ }^{1}$ Frank J. Bellafiore, MD, ${ }^{2}$ Muhamad A. Amine, MD, ${ }^{3}$ and Wael Hassaneen, MD, PhD ${ }^{1,4}$ \\ ${ }^{1}$ Carle Illinois College of Medicine, University of Illinois Urbana-Champaign, Champaign, Illinois; and Departments of ${ }^{2}$ Pathology, ${ }^{3}$ Otolaryngology, Head and Neck Surgery, and \\ ${ }^{4}$ Neurosurgery, Carle Foundation Hospital, Champaign, Illinois
}

\begin{abstract}
BACKGROUND Allergic fungal sinusitis (AFS) is an immunoglobulin E-mediated reaction to fungal organisms in the sinonasal region and can be categorized as acute or chronic. Acute infection is typical in immunocompromised patients, while chronic infection is classically seen in immunocompetent patients. Spread of infection to the skull base is a rare and potentially lethal complication of prolonged infection. Surgical management is frequently augmented with steroid therapy to prevent recurrence.

OBSERVATIONS The authors present a case of a 20 -year-old African American male with prolonged headaches and blurred vision who was diagnosed with chronic invasive fungal sinusitis resulting in invasion of fungal burden into the anterior skull base and the posterior aspect of the clivus, in addition to complete obliteration of the maxillary sinus. The patient was managed surgically without complication and with gradual improvement in vision.
\end{abstract}

LESSONS Early management and detection of AFS should be a focus to prevent erosion of the fungal burden into the skull base. Neurosurgery and ear, nose, and throat surgery have a multidisciplinary role in the management of advanced AFS cases.

https://thejns.org/doi/abs/10.3171/CASE2161

KEYWORDS allergic fungal sinusitis; skull base surgery; invasive infection; aspergillosis

Allergic fungal sinusitis (AFS) is an immunoglobulin E-mediated reaction to fungal organisms in the sinonasal region. It is thought to represent $12 \%$ of all cases of sinusitis. ${ }^{1}$ In some cases, it can become a potentially fatal infection resulting in invasion and destruction of nasal and cranial structures. This can occur acutely or chronically. Acute invasive fungal sinusitis is known to occur more commonly in immunocompromised patients, with fatality rates between $50 \%$ and $80 \%{ }^{2}$ Chronic invasive fungal sinusitis (CIFS) is thought to classically infect the immunocompetent host and is frequently diagnosed late in its course because of nonspecific symptoms. ${ }^{3}$

While risk factors unique to AFS are largely unknown, some studies indicate that smoking may lead to increased risk. ${ }^{4}$ Diabetes, hypertension, and asthma have also been associated with AFS. ${ }^{1}$ Additionally, African American patients, patients in rural counties with diminished access to primary care, and patients with older and poorer-quality homes were found to have increased rates of bone erosion in the setting of AFS, a known finding of CIFS. ${ }^{5,6}$
Patients diagnosed with CIFS typically present with chronic rhinosinusitis unresponsive to conservative management, congestion, and chronic headaches. ${ }^{1,7}$ The Bent-Kuhn diagnostic criteria for AFS are widely accepted, which include type 1 hypersensitivity, nasal polyposis, characteristic computed tomography (CT) findings, eosinophilic mucus without fungal invasion into sinus tissue, and positive fungal staining of evacuated sinus contents. ${ }^{8}$ Erosion into bone structures can also be seen in $20 \%-25 \%$ of patients. ${ }^{7,9}$ CT findings often help to localize the infection and show opacification of sinuses, remodeling, thinning, and erosion of bony sinus walls. ${ }^{10}$ While the infection can infect multiple sinuses, the ethmoid sinus was found to be the most commonly infected. ${ }^{11}$ Spread of infection to the skull base is very rare and potentially lethal. ${ }^{12}$ Treatment largely involves the use of steroids and potentially antifungal medication, in addition to surgical debridement of the fungal mass. ${ }^{13-15}$

Few studies have described AFS, notably CIFS, to invade the skull base, resulting in complete obliteration of the bony sinus wall.

ABBREVIATIONS AFS = allergic fungal sinusitis; CIFS = chronic invasive fungal sinusitis; $\mathrm{CT}=$ computed tomography; ENT = ear, nose, and throat; MRI = magnetic resonance imaging.

INCLUDE WHEN CITING Published April 19, 2021; DOI: 10.3171/CASE2161.

SUBMITTED January 28, 2021. ACCEPTED February 1, 2021.

(C) 2021 The authors, CC BY-NC-ND 4.0 (http://creativecommons.org/licenses/by-nc-nd/4.0/). 

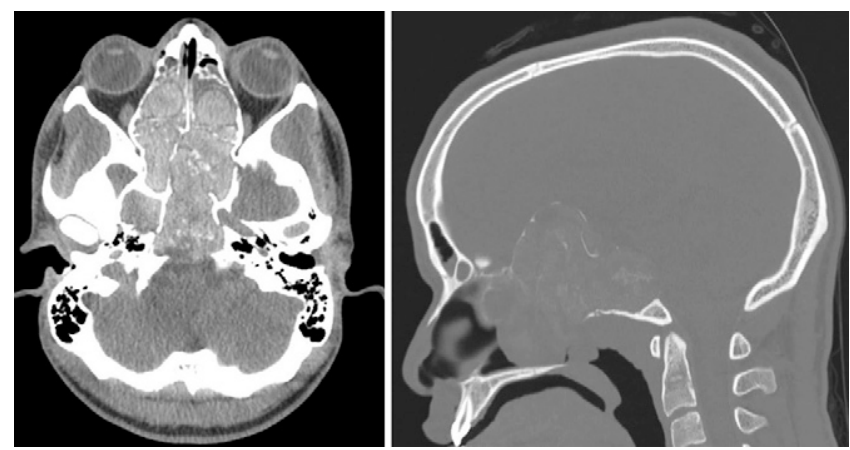

FIG. 1. Preoperative axial (left) and sagittal (right) CT demonstrating extensive fungal burden and opacification of sinuses.

In this report, we present a case of CIFS with invasion of the skull base that resulted in the complete obliteration of the maxillary sinus. Patient presentation, management, outcomes, and lessons learned are discussed, along with a review of studies that document invasion of AFS into the skull base.

\section{Illustrative Case}

The patient was an immunocompetent 20-year-old African American male with no significant past medical history. He presented to the clinic following a minor head trauma that precipitated abnormal eye movement in his left eye. His history and physical examination revealed a 6th cranial nerve palsy and a 2-year history of progressive loss of visual field in the left eye and a visual acuity of $20 / 800$. His right eye had normal extraocular movement and 20/20 acuity. The patient also reported progressive congestion and rhinorrhea in his nose alongside the course of his left-eye visual loss. Nasal endoscopy revealed extensive polyps and an identifiable mass. The patient was started on dexamethasone and famotidine to minimize inflammation and curb the allergic response preoperatively.

CT revealed a hyperdense mass with scattered calcifications centered in the paranasal sinuses with either severe remodeling or disruption, and extension through the floor of the anterior cranial fossa was found (Fig. 1). The mass was seen to extend into both the right and left middle cranial fossae, the sella turcica, the left orbit apex, the left orbital superior fissure, and possibly the left optic canal. The mass also extended along the posterior aspect of the clivus, also bulging into the prepontine cistern. On magnetic resonance imaging (MRI), the fungal mass exhibited a heterogeneous appearance, including intrinsic T1 hyperintensity, prominent T2 hypointensity, and peripheral areas of enhancement (Fig. 2).

An expanded endoscopic endonasal resection of the mass was performed by the skull base team, including otolaryngology and neurosurgery. Epinephrine was first used to decongest the nasal cavity, which revealed extensive polypoid disease. The concha bullae were incised bilaterally. The left side was inundated with fungus, which was removed with careful microdissection, irrigation, and suction. The middle turbinate was resected. The uncinate process was removed to gain access to the maxillary sinus, which was full of secretions. The anterior and posterior ethmoid cells and Haller cells were also filled with a thick, brownish fungal burden. Once the sphenoid sinus was reached and found also to be filled with fungus, the fungal burden was resected. The fungus invaded and eroded a large area of the skull from the anterior skull base to the clivus, creating many pockets of fungus that were resected with careful microdissection, irrigation, and suction. An external ventricular drainage catheter was used to irrigate difficult-to-reach pockets. No dural invasion was noted, with no cerebrospinal fluid leak encountered during the procedure. The surgery took over 8 hours to fully remove the fungal invasion. Figure 3 shows a procedural image in addition to microscopic and histological evaluation of samples collected. The microscopic findings of the fungus and mucus demonstrated evidence of laminated allergic mucin with eosinophil debris and Charcot-Leyden crystals, in addition to degenerating sinus tissue and bone. Septate hyphae were also observable on Grocott methenamine silver stains. Cultures obtained from the fungal burden indicated growth of Aspergillus spp. and Curvularia spp.

Figure 4 shows postoperative resolution of the fungal burden on MRI. Postoperatively, the 6th cranial nerve palsy had resolved, and the patient reported improvement in his vision. Dexamethasone was tapered and transitioned to a betamethasone dipropionate nasal rinse. No systemic antifungal therapy was used. At the patient's last neuro-ophthalmology follow-up, the visual acuity in his left eye had improved to 20/500. He still had congestion and swelling, which were treated with a triamcinolone acetonide injection and continued use of the steroid rinse. On MRI, resolution of the fungal mass was shown, but the right maxillary sinus was obliterated. At the 18-month follow-
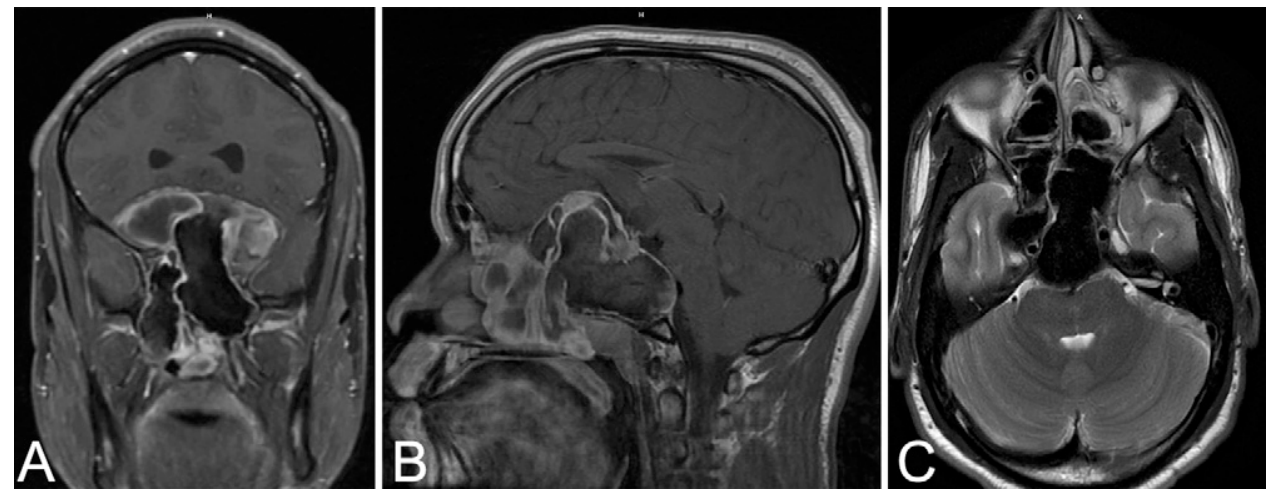

FIG. 2. Preoperative MRI. A: Coronal T1-weighted image. B: Sagittal T1-weighted image. C: Axial T2weighted image. 

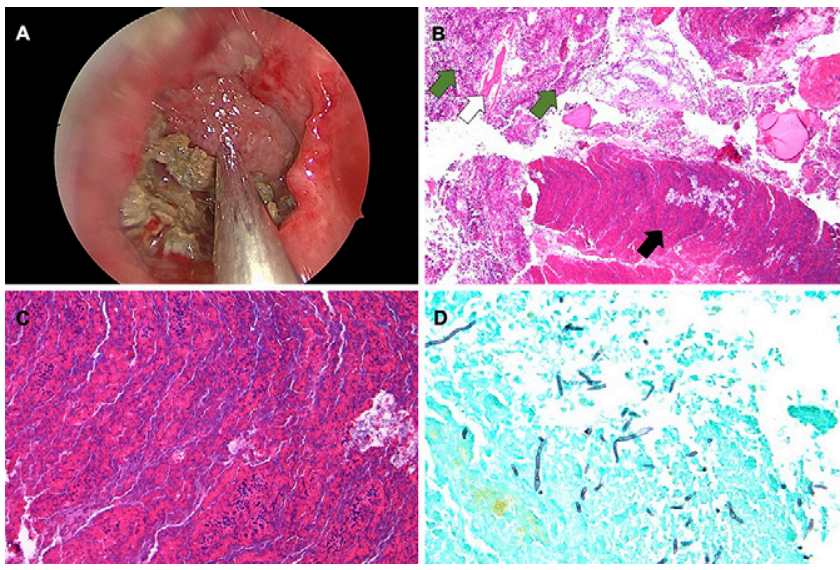

FIG. 3. Intraoperative image of fungal burden and pathological sections. A: Endoscopic debridement of extradural fungal burden. B: Laminated allergic mucin, with layers of eosinophil debris and Charcot-Leyden crystals (black arrow). Reactive degenerated bone (white arrow) and degenerative sinus tissue (green arrows) can also be observed. Original magnification $\times 40$. C: Higher magnification of laminated allergic mucin. Original magnification $\times 200$. D: Septate $45^{\circ}$ hyphae visible on a Grocott methenamine silver stain. Original magnification $\times 200$.

up, the patient reported stable vision in his left eye, which allowed continuation of his activities of daily living, including sports.

\section{Discussion}

\section{Observations}

AFS can present acutely, such as in immunocompromised patients, and chronically, typically in immunocompetent patients. ${ }^{2,3}$ Common organisms implicated in AFS include Aspergillus, Mucor, and Curvularia spp., ${ }^{15}$ with Curvularia remaining a rare fungus associated with warm, humid climates. ${ }^{16}$ Our patient also reported various nonspecific symptoms, such as congestion, headache, and alterations in vision, 2 years before his diagnosis and surgical treatment. Incidental imaging after minor head trauma revealed findings to warrant further investigation. Because of the common and nonspecific symptoms frequently reported by patients before diagnosis, CIFS is often diagnosed late in the course of infection. ${ }^{1,3}$

Despite the documented delay in the diagnosis of CIFS, the spread of the infection to the skull base is considered very severe and is rare. In this report, we describe the presentation of CIFS in a 20 -year-old immunocompetent African American male. The fungal invasion obliterated the maxillary sinus wall and spread from the anterior skull base to the clivus. The patient's ocular symptoms may have been caused by compressive effects of the fungal mass, which has previously been documented in rare cases. ${ }^{17}$

Very few cases of skull base invasion from AFS have been described in the literature. Table 1 summarizes all case reports and case series we could find that describe such a degree of invasion for AFS. As in this case, infection does not typically spread into the dura. In a case report by Meccariello et al., a 48-year-old female was found to have extensive fungal disease in all paranasal sinuses involving the medial orbital wall and anterior skull base invasion, including erosion of the cribriform lamina and clivus, in addition to a dehiscence proximal to the right internal carotid artery and optic
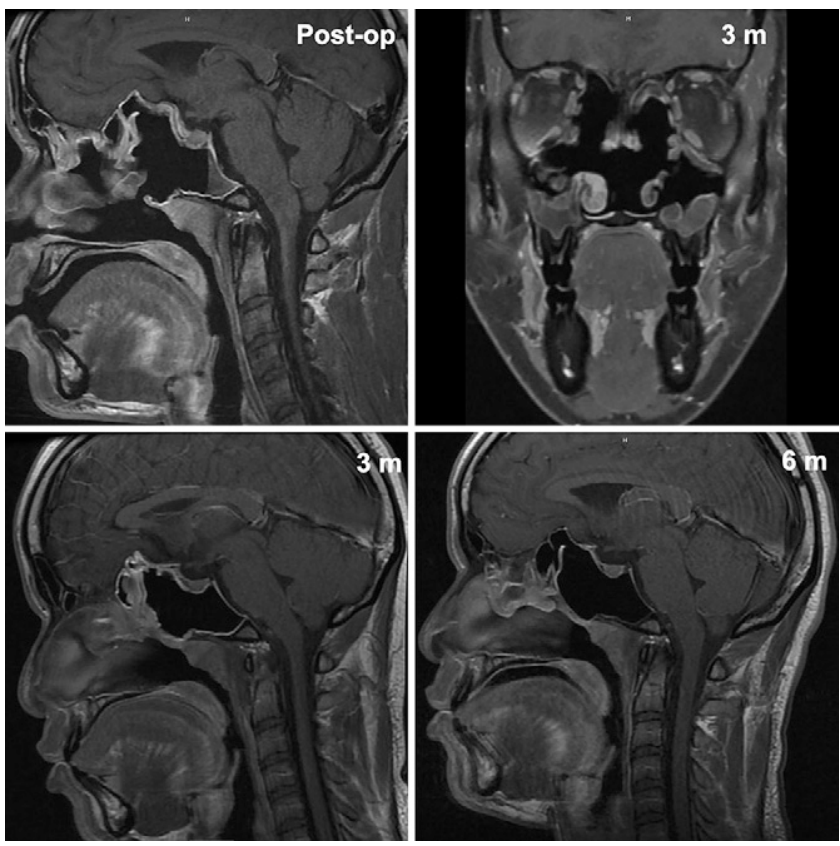

FIG. 4. Follow-up MRI at immediate post-operative period, and at 3 months $(3 \mathrm{~m})$ and 6 months $(6 \mathrm{~m})$ post-operatively showing resolution of the fungal burden.

nerve. ${ }^{18}$ While encasement of the carotid artery was not observed in our case, the extension of the fungal burden was similar in that the mass began to protrude into the prepontine cistern and posterior aspect of the clivus. A case series by Ikram et al. presented 26 patients with intracranial extension of AFS, including 1 patient with invasion into the anterior skull base and bilateral orbits. ${ }^{19}$ All cases described extradural fungal invasion only. In one case report, however, an abscess was observed, necessitating drainage via a right temporal craniotomy. ${ }^{20}$ The potential for observed intracerebral extension and comfort around the skull base highlights the need for interdisciplinary management teams that include neurosurgery and ear, nose, and throat (ENT) surgery to manage AFS patients with skull base invasion. ${ }^{21,22}$

In cases of AFS with intracranial extension of the fungal burden, surgical debridement and medical management can often result in complete resolution of symptoms. ${ }^{12,19}$ In some cases, untreated AFS can result in permanent damage to the optic nerve and surrounding ocular structures, resulting in vision loss, even after treatment. $^{23}$ Extended follow-up may be required to appreciate the gradual return of function, if any, in vision in patients with CIFS. Furthermore, recurrence is also a significant challenge, reported to be as high as $100 \%$ in some studies in which only surgical debridement was performed. ${ }^{15}$ To reduce rates of complications and recurrence, steroid therapy can be used to augment surgical management of fungal invasion. ${ }^{13,15}$ In addition, while the benefit of antifungal medications has not been completely elucidated, some clinical trials demonstrate their utility in improving radiographical findings in patients. $^{14}$

\section{Lessons}

In summary, while AFS is a relatively common infectious process, it rarely is observed to invade the skull base and result in the 
TABLE 1. Relevant case reports and case series

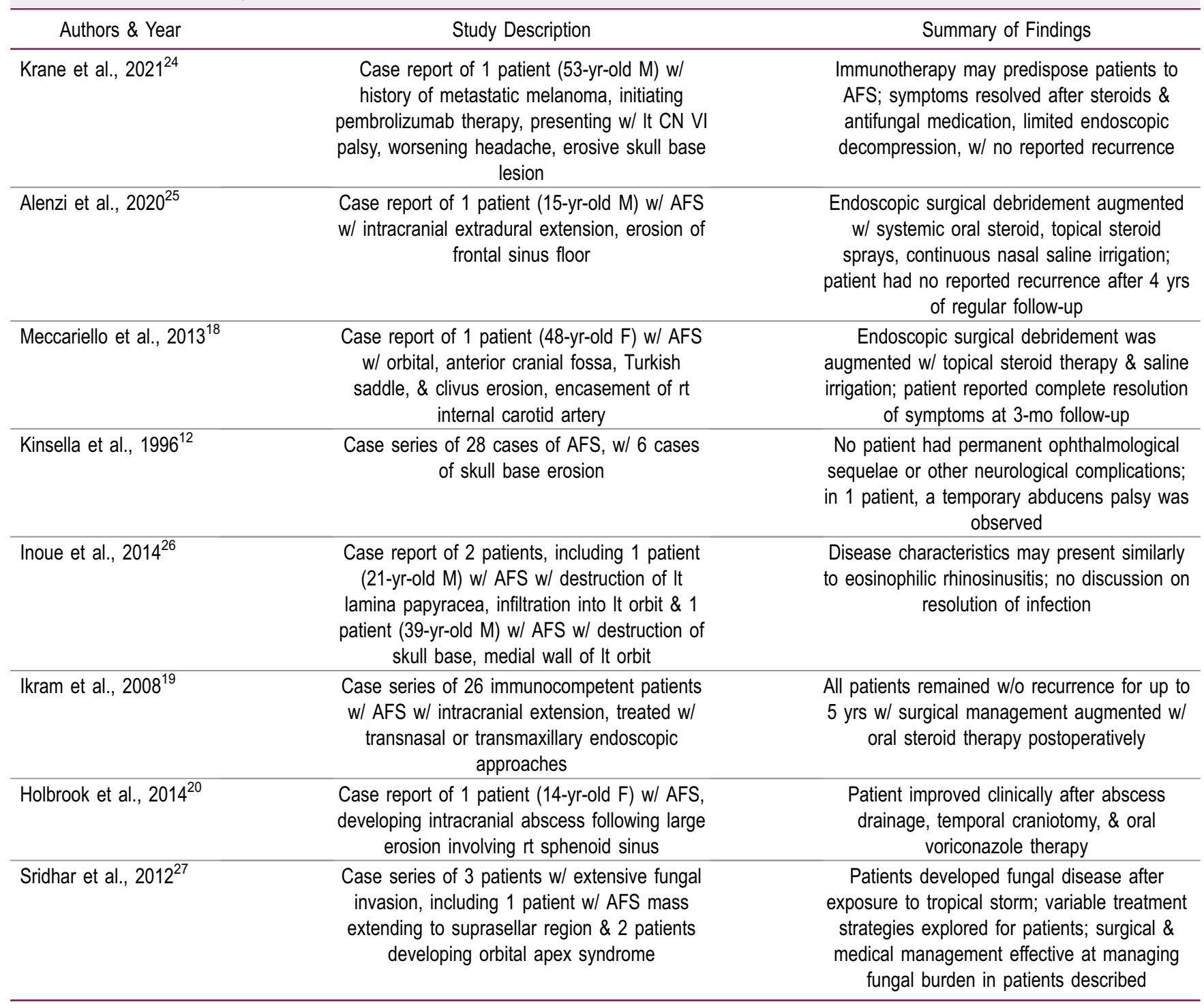

$\mathrm{CN}=$ cranial nerve.

obliteration of a sinus wall. In this case, there was no intradural involvement; however, abscess resulting from AFS has also been previously reported. Early diagnosis of AFS should be a priority in the future to prevent such advanced presentations of the infective process. We believe that neurosurgery and ENT surgery have an important multidisciplinary role in the surgical management of AFS, notably CIFS, as evidenced by this case.

\section{References}

1. Salamah MA, Alsarraj M, Alsolami N, et al. Clinical, radiological, and histopathological patterns of allergic fungal sinusitis: a singlecenter retrospective study. Cureus. 2020;12(7):e9233.

2. Aribandi M, McCoy VA, Bazan C III. Imaging features of invasive and noninvasive fungal sinusitis: a review. Radiographics. 2007;27(5):1283-1296.

3. Alotaibi NH, Omar OA, Altahan M, et al. Chronic invasive fungal rhinosinusitis in immunocompetent patients: a retrospective chart review. Front Surg. 2020;7:608342.
4. Hutson K, Clark A, Hopkins $C$, et al. Evaluation of smoking as a modifying factor in chronic rhinosinusitis. JAMA Otolaryngol Head Neck Surg. 2021;147(2):159-165.

5. Miller JD, Deal AM, McKinney KA, et al. Markers of disease severity and socioeconomic factors in allergic fungal rhinosinusitis. Int Forum Allergy Rhinol. 2014;4(4):272-279.

6. Wise SK, Ghegan MD, Gorham E, Schlosser RJ. Socioeconomic factors in the diagnosis of allergic fungal rhinosinusitis. Otolaryngol Head Neck Surg. 2008;138(1):38-42.

7. McClay JE, Marple B, Kapadia L, et al. Clinical presentation of allergic fungal sinusitis in children. Laryngoscope. 2002;112(3):565-569.

8. Bent JP III, Kuhn FA. Diagnosis of allergic fungal sinusitis. Otolaryngol Head Neck Surg. 1994;111(5):580-588.

9. Nussenbaum B, Marple BF, Schwade ND. Characteristics of bony erosion in allergic fungal rhinosinusitis. Otolaryngol Head Neck Surg. 2001;124(2):150-154.

10. Mukherji SK, Figueroa RE, Ginsberg LE, et al. Allergic fungal sinusitis: CT findings. Radiology. 1998;207(2):417-422. 
11. Gupta AK, Ghosh S, Gupta AK. Sinonasal aspergillosis in immunocompetent Indian children: an eight-year experience. Mycoses. 2003;46(11-12):455-461.

12. Kinsella JB, Rassekh $\mathrm{CH}$, Bradfield JL, et al. Allergic fungal sinusitis with cranial base erosion. Head Neck. 1996;18(3):211-217.

13. Marple BF. Allergic fungal rhinosinusitis: current theories and management strategies. Laryngoscope. 2001;111(6):1006-1019.

14. Ponikau JU, Sherris DA, Weaver A, Kita H. Treatment of chronic rhinosinusitis with intranasal amphotericin $\mathrm{B}$ : a randomized, placebo-controlled, double-blind pilot trial. J Allergy Clin Immunol. 2005;115(1):125-131.

15. Schubert MS. Allergic fungal sinusitis: pathophysiology, diagnosis and management. Med Mycol. 2009;47(suppl 1):S324-S330.

16. Edelmayer $\mathrm{L}$, Ito $\mathrm{C}$, Lee WS, et al. Conversion to chronic invasive fungal sinusitis from allergic fungal sinusitis in immunocompetence. Laryngoscope. 2019;129(11):2447-2450.

17. El Mograbi A, Soudry E. Ocular cranial nerve palsies secondary to sphenoid sinusitis. World J Otorhinolaryngol Head Neck Surg. 2017;3(1):49-53.

18. Meccariello G, Deganello A, Mannelli G, et al. Allergic fungal rhinosinusitis infiltrating anterior skull base and clivus. Auris Nasus Larynx. 2013;40(4):405-408.

19. Ikram M, Akhtar S, Ghaffar S, Enam SA. Management of allergic fungal sinusitis with intracranial spread. Eur Arch Otorhinolaryngol. 2008;265(2):179-184.

20. Holbrook JF, Eastwood JD, Kilani RK. Intracranial abscess as a complication of allergic fungal sinusitis. J Neuroimaging. 2014;24(1): 95-98.

21. Makhdoom N, Balkheur K, Foda MA, et al. Fungal sinusitis with intracranial extension in immuno-competent patients: surgical planning according to relation to eloquent neurovascular structures. J Taibah Univ Med Sci. 2008;3(1):33-43.

22. Liu JK, Schaefer SD, Moscatello AL, Couldwell WT. Neurosurgical implications of allergic fungal sinusitis. J Neurosurg. 2004;100(5): 883-890.
23. Vashishth A. Extensive allergic fungal rhinosinusitis: ophthalmic and skull base complications. Indian J Otolaryngol Head Neck Surg. 2015;67(3):227-233.

24. Krane NA, Beswick DM, Sauer D, et al. Allergic fungal sinusitis imitating an aggressive skull base lesion in the setting of pembrolizumab immunotherapy. Ann Otol Rhinol Laryngol. 2021; 130(1):108-111.

25. Alenzi HL, Al Momen A, Molani F. Pediatric allergic fungal rhinosinusitis with extensive intracranial extension-case report and literature review. Int J Surg Case Rep. 2020;75:437-440.

26. Inoue T, Kamijo A, Nakajima M, et al. Two cases of allergic fungal rhinosinusitis with bone destruction. Article in Japanese. Arerugi. 2014;63(7):951-957.

27. Sridhar J, Lam BL, Pasol J, Sternau L. Neuro-ophthalmic manifestations of fungal disease associated with posthurricane environment. J Neuroophthalmol. 2012;32(3):197-201.

\section{Disclosures}

The authors report no conflict of interest concerning the materials or methods used in this study or the findings specified in this paper.

\section{Author Contributions}

Conception and design: Hassaneen, Naik, Yang, Amine. Acquisition of data: Hassaneen, Bellafiore. Analysis and interpretation of data: Naik, Yang, Bellafiore. Drafting the article: Hassaneen, Naik, Yang. Critically revising the article: Hassaneen, Naik. Reviewed submitted version of manuscript: Hassaneen, Naik, Yang, Amine. Administrative/technical/ material support: Naik. Study supervision: Hassaneen. Operating surgeon: Amine.

\section{Correspondence}

Wael Hassaneen: Carle Foundation Hospital, Champaign, IL. wael.mostafa@carle.com. 